\title{
A Characterization of Normal Matrices ${ }^{1}$
}

\author{
Alan J. Hoffman and Olga Taussky
}

\begin{abstract}
A matrix $A$ is called normal if $A A^{*}=A^{*} A$, where $A^{*}$ is the transposed and conjugate matrix of $A$. It is known that for a pair of commuting matrices, $A, B$, there exists an ordering of the characteristic roots $\alpha_{1}, \ldots, \alpha_{n}$ of $A$ and $\beta_{1}, \ldots, \beta_{n}$ of $B$, such that every polynomial $p(A, B)$ has as characteristic roots the numbers $p\left(\alpha_{i}, \beta_{i}\right)$. This property is, in general, weaker than commutativity, but does imply it if $B=A^{*}$. It is shown that this property already implies commutativity of $A$ and $A *$ if it is assumed to hold for only one polynomial, provided the latter is suitably chosen. Polynomials of first and second degree are examined for their suitability.
\end{abstract}

Normal matrices can be characterized in many ways. In the original definition $[1]^{2}$ a matrix $A$ is called normal if $A A^{*}=A^{*} A$, where $A^{*}$ is the transposed and conjugate matrix of $A$. It is known that for a pair of commuting matrices $A, B$, there exists an ordering of the characteristic roots $\alpha_{1}, \ldots, \alpha_{n}$ of $A$ and $\beta_{1}, \ldots, \beta_{n}$ of $B$, such that every polynomial $p(A, B$,$) has as characteristic roots the$ numbers $p\left(\alpha_{i}, \beta_{i}\right)$, see [2]. This property is, in general, weaker than commutativity, but does imply it if $B=A^{*}$. This follows from the fact that two matrices with this property can be transformed to upper triangular form simultaneously by a unitary similarity transformation [3]. Even if the property is assumed only for linear polynomials $p\left(A, A^{*}\right)$, commutativity follows. This is a consequence of the fact that $A$ is the sum of two normal matrices,

$$
A=\frac{A+A^{*}}{2}+\frac{A-A^{*}}{2} \text {. }
$$

For the matrices $\left(A+A^{*}\right) / 2,\left(A-A^{*}\right) / 2$ will again have the above property with respect to linear polynomials; this, however, implies that they commute $[4,5]$. Hence $A$ and $A^{*}$ commute.

It will now be shown that the above property already implies the commutativity of $A$ and $A^{*}$ if it is assumed to hold for only one polynomial, provided the latter is suitably chosen. Two special results in this direction were obtained earlier [6]; the polynomials considered then were $A+A^{*}$ and $A A^{*}$, and it was assumed that the special ordering of the characteristic roots implied that $\beta_{i}=\bar{\alpha}_{i}$.

Certain polynomials of first and second degree are examined for their suitability. The result is that any polynomial of first degree is suitable, while not every polynomial of second degree is, although a large class among them is suitable.

In what follows we assume that $A=\left(a_{i k}\right)$ is an $n \times n$ matrix with complex numbers as elements.

TheOREm 1. If $\alpha_{1}, \ldots, \alpha_{n}$ are the eigenvalues of an $n \times n$ matrix $A, \alpha$ and $\beta$ are two complex numbers different from 0 , and the eigenvalues of $\alpha A+\beta A^{*}$ are

1 The preparation of this paper was sponsored (in part) by the Office of Scientific Research, United States Air Force.

${ }^{2}$ Figures in brackets indicate the literature references at the end of this paper. $\alpha \alpha_{i}+\beta \bar{\alpha}_{P i}$, where $P$ is a permutation of the integers $1, . . ., n$, then $A$ is normal.

Proof. It is clearly no restriction to assume $\beta=1$. Further, since the hypothesis and conclusion of the theorem are concerned with eigenvalues of an operator $A$ in unitary $n$-space, we may assume that an orthonormal basis has been chosen in the unitary $n$-space, so that the matrix $A$ is (upper) triangular; i. e., $a_{i k}=0$ for $i>k$, and $a_{i i}=\alpha_{i}$. We shall show that

$$
a_{i k}=0 \quad \text { for } i \neq k,
$$

which implies that $A$ is normal.

The argument is based on the fact that the second elementary symmetric function $S_{2}$ of the eigenvalues $\gamma_{1}, \ldots, \gamma_{n}$ of $\alpha A+A^{*}$ may be computed in two ways. First, since

we have

$$
\gamma_{i}=\alpha \alpha_{i}+\bar{\alpha}_{P i} \quad(i=1, \ldots, n),
$$

$$
2 S_{2}=\sum_{i=1}^{n} \sum_{k \neq i}\left[\alpha^{2} \alpha_{i} \alpha_{k}+\bar{\alpha}_{P i} \bar{\alpha}_{P k}+\alpha\left(\alpha_{i} \bar{\alpha}_{P k}+\bar{\alpha}_{P i} \alpha_{k}\right)\right] .
$$

Second, since $S_{2}$ is also the sum of all principal $2 \times 2$ minors $M_{i k}$ of $\alpha A+A^{*}$, we have

$$
\begin{aligned}
2 S_{2}=\sum_{i=1}^{n} \sum_{k \neq i} M_{i k}=\sum_{i=1}^{n} \sum_{k \neq i} & {\left[\alpha^{2} \alpha_{i} \alpha_{k}+\bar{\alpha}_{i} \bar{\alpha}_{k}\right.} \\
& \left.+\alpha\left(\alpha_{i} \bar{\alpha}_{k}+\bar{\alpha}_{i} \alpha_{k}-a_{i k} \bar{a}_{i k}\right)\right] .
\end{aligned}
$$

As $i$ and $k$ range over all possible distinct ordered pairs of elements of the set $\{1, \ldots, n\}$, so do $P_{i}$ and $P_{k}$. Hence if we subtract (3) from (2), we obtain

$$
\sum_{i=1}^{n} \sum_{k \neq i}\left(\alpha_{i} \bar{\alpha}_{P k}+\bar{\alpha}_{P i} \alpha_{k}-\alpha_{i} \bar{\alpha}_{k}-\bar{\alpha}_{i} \alpha_{k}+a_{i k} \bar{a}_{i k}\right)=0 .
$$

But $a_{i k} \bar{a}_{i k} \geq 0$. Therefore, if we show that

$$
R \sum_{i=1}^{n} \sum_{k \neq i}\left(\alpha_{i} \bar{\alpha}_{P k}+\bar{\alpha}_{P i} \alpha_{k}-\alpha_{i} \bar{\alpha}_{k}-\bar{\alpha}_{i} \alpha_{k}\right) \geqq 0,
$$

then (4) will imply (1). 
Write

$$
\alpha_{j}=\lambda_{j}+i \mu_{j} \quad(j=1, \ldots, n),
$$

where $\lambda_{j}$ and $\mu_{j}$ are real.

Then (5) becomes

$$
2 \sum_{i=1}^{n} \sum_{k \neq i}\left[\lambda_{i}\left(\lambda_{P k}-\lambda_{k}\right)+\mu_{i}\left(\mu_{P k}-\mu_{k}\right)\right] \geq 0 .
$$

We shall prove

$$
\sum_{i=1}^{n} \sum_{k \neq i} \lambda_{i}\left(\lambda_{P k}-\lambda_{k}\right) \geq 0 .
$$

The same argument will apply to the $\mu$ 's, and hence (6) will be established. Now for fixed $i$,

$$
\sum_{k \neq i} \lambda_{i}\left(\lambda_{P k}-\lambda_{k}\right)=\lambda_{i} \sum_{k \neq i}\left(\lambda_{P k}-\lambda_{k}\right)=\lambda_{i}\left(\lambda_{i}-\lambda_{P i}\right) .
$$

Hence (7) is equivalent to

$$
\sum_{i=1}^{n} \lambda_{i}\left(\lambda_{i}-\lambda_{P i}\right) \geq 0 .
$$

Inequality (8) is a special case of a known inequality ([7], theorem 368), which states that if $a_{1} \geq \ldots \geq a_{n}, b_{1} \geq \ldots \geq b_{n}$, and $P$ is any permutation of $\{1, \ldots, n\}$, then

$$
\sum a_{i} b_{i} \geq \sum a_{i} b_{P i} .
$$

Set $a_{i}=b_{i}=\lambda_{i}$, and (8) is an immediate consequence of $(9)$.

Theorem 2. ${ }^{3}$ If $\alpha_{1}, \ldots . \alpha_{n}$ are the eigenvalues of an $n \times n$ matrix $A$, and the eigenvalues of $A A^{*}$ are $\alpha_{1} \bar{\alpha}_{P 1}, \ldots, \alpha_{n} \bar{\alpha}_{P n}$, where $P$ is a permutation of the integers 1,...., $n$, then $A$ is normal.

Proof. As in theorem 1, we may assume $A$ is upper triangular. We shall compute trace $A A^{*}$ in two ways. First, since trace $A A^{*}$ is the sum of the eigenvalues of $A A^{*}$, we have by hypothesis

$$
\text { trace } A A^{*}=\sum_{i=1}^{n} \alpha_{i} \bar{\alpha}_{P i}, \quad \alpha_{i} \bar{\alpha}_{P i} \geq 0
$$

Second, since trace $A A^{*}$ is the sum of the elements on the diagonal of $A A^{*}$, we have

$$
\operatorname{trace} A A^{*}=\sum_{i=1}^{n} \alpha_{i} \bar{\alpha}_{i}+\sum_{i=1}^{n} \sum_{k \neq i} a_{i k} \bar{a}_{i k} .
$$

Write $\alpha_{j}=\rho_{j} e^{i \theta j}{ }_{j}$ where $\rho_{j} \geq 0$. We have, from (11) and (10),

$$
\sum_{i=1}^{n} \rho_{1}^{2}+\sum_{i=1}^{n} \sum_{k \neq i}^{n} a_{i k} \bar{a}_{i k}=\sum_{i=1}^{n} \rho_{i} \rho_{P i} .
$$

But from inequality (8), we see that

$$
\sum_{i=1}^{n} \rho_{1}^{2} \geq \sum_{i=1}^{n} \rho_{i} \rho_{P i}
$$

3 This theorem can also be proved from known inequalities, see [8], between the eigenvalues of a matrix $A$ and the eigenvalues of $A A^{*}$.

Since $a_{i k} \bar{a}_{i k} \geq 0$, the only way for (12) and (13) to be consistent is for

$$
a_{i k} \bar{a}_{i k}=0 \quad \text { for all } i \neq k \text {; }
$$

i. e., $A$ is normal.

One can use essentially the same argument to establish

Theorem 3. Let $\alpha_{1}, \ldots, \alpha_{n}$ be the eigenvalues of an $n \times n$ matrix $A, P$ a permutation of the integers $1, \ldots, n$, and $a_{1}, a_{2}, b_{1}, b_{2}, c_{1}, c_{2}$ complex numbers. Let the eigenvalues of

$$
a_{1} A+a_{2} A^{*}+b_{1} A^{2}+b_{2} A^{* 2}+c_{1} A A^{*}+c_{2} A^{*} A
$$

be

$a_{1} \alpha_{i}+a_{2} \bar{\alpha}_{P i}+b_{1} \alpha_{i}^{2}+b_{2} \bar{\alpha}_{P i}^{2}+c_{1} \alpha_{i} \bar{\alpha}_{P i}$

If, in addition

$$
+c_{2} \bar{\alpha}_{P i} \alpha_{i} \quad(i=1, \ldots, n) .
$$

then $A$ is normal.

$$
c_{1}+c_{2} \neq 0 \text {, }
$$

What if condition (15) is omitted; i. e., if $c_{1}=c_{2}$ ? Then theorem 3 may not hold. There are two cases to consider:

$$
\text { (i) } c_{1}=0 \text {, (ii) } c_{1} \neq 0 \text {. }
$$

In case (i), if $b_{1}=b_{2}=0$, then the theorem reduces to theorem 1 , so $A$ is normal (unless $a_{1} a_{2}=0$, where the theorem fails). The theorem fails unless $b_{1}=b_{2}=0$. A counterexample is furnished by

$$
\left(\begin{array}{ll}
c & d \\
0 & 0
\end{array}\right),
$$

where $d \neq 0$ is arbitrary, $c=-a_{1} / b_{1}$ (if $b_{1} \neq 0$ ), or $\bar{c}=-a_{2} / b_{2}$ (if $b_{2} \neq 0$ ).

For case (ii), the situation is somewhat more complicated. For example, if $a_{1} a_{2}$ is real and negative, the theorem fails, as one can see from the counterexample

$$
\left(\begin{array}{ll}
0 & a \\
0 & 0
\end{array}\right),
$$

where $a^{2} c_{1}^{2}=-a_{1} a_{2}$

On the other hand, if (14) is formally Hermitian, the theorem does hold. Specifically, we have

Theorem 4. Let $\alpha_{1}, \ldots, \alpha_{n}$ be the eigenvalues of an $n \times n$ matrix $A$; let $P$ be a permutation of the integers $1, \ldots, n ; a, b$ complex numbers, $c$, d real numbers not both 0 ; let the eigenvalues of

$$
\begin{aligned}
& a A+\bar{a} A^{*}+b A^{2}+\bar{b} A^{* 2}+c A A^{*} \\
& +d A^{*} A(c, d \text { real, not both } 0) \\
& \text { be } \\
& a \alpha_{i}+\bar{a} \bar{\alpha}_{P i}+b \alpha_{i}^{2}+\bar{b} \bar{\alpha}_{P i}^{2}+c \alpha_{i} \bar{\alpha}_{P i} \\
& \text { then } A \text { is normal. } \\
& +d \bar{\alpha}_{P i} \alpha_{i} \quad(i=1, \ldots, n) ;
\end{aligned}
$$


Proof. If $c \neq-d$, the situation is covered by theorem 3. If $c=-d$, it is clearly no loss of generality to take $c=1, d=-1$. Now order the eigenvalues of $A$, so that

$$
R\left(a \alpha_{1}+b \alpha_{1}^{2}\right) \geq \ldots . \geq R\left(a \alpha_{n}+b \alpha_{n}^{2}\right) .
$$

We may assume that $A$ is upper triangular, and that the eigenvalues of $A$ appear on the main diagonal in the order $\alpha_{1}, \alpha_{2}, \ldots, \alpha_{n}$.

Then let $B$ stand for the Hermitian matrix (16), and denote its eigenvalues by $\beta_{1} \geq \ldots \geq \beta_{n}$.

It has been shown ( [9], theorem I) that

$$
\begin{gathered}
b_{11} \leq \beta_{1} \\
b_{11}+b_{22} \leq \beta_{1}+\beta_{2} \\
\cdot \\
\cdot \\
\cdot \\
b_{11}+\ldots+b_{n-1, n-1} \leq \beta_{1}+\ldots+\beta_{n-1} .
\end{gathered}
$$

But

$$
b_{11}=2 R\left(a \alpha_{1}+b \alpha_{1}^{2}\right)+\sum_{k=2}^{n} a_{1 k} \bar{a}_{1 k},
$$

and

$$
\beta_{1}=\max \left[R\left(a \alpha_{i}+\overline{a \alpha}_{P i}+b \alpha_{i}^{2}+\bar{b}_{P i}^{2}\right)\right] \leq 2 R\left(a \alpha_{1}+b \alpha_{1}^{2}\right),
$$

by (17). Since $a_{1 k} \bar{a}_{1 k} \geq 0 \quad(k=2, \ldots, n)$, the only way for (19) and (20) to be consistent with the first inequality of (18) is if

$$
a_{i k}=0 \quad(k=2, \ldots, n) .
$$

Now, in view of (21), it follows that

$$
b_{11}+b_{22}=2 R\left[a\left(\alpha_{1}+\alpha_{2}\right)+b\left(\alpha_{1}^{2}+\alpha_{2}^{2}\right)\right]+\sum_{k=3}^{n} a_{2 k} \bar{a}_{2 k},
$$

and

$$
\begin{aligned}
\beta_{1}+\beta_{2} & =\max _{i \neq j} R\left\{a\left(\alpha_{i}+\alpha_{j}\right)+\bar{a}\left(\bar{\alpha}_{P i}+\bar{\alpha}_{P j}\right.\right. \\
& \left.+b\left(\alpha_{i}^{2}+\alpha_{j}^{2}\right)+\bar{b}\left(\bar{\alpha}_{P i}^{2}+\bar{\alpha}_{P j}^{2}\right)\right\} \\
& \leq 2 R\left[a\left(\alpha_{1}+\alpha_{2}\right)+b\left(\alpha_{1}^{2}+\alpha_{2}^{2}\right)\right] .
\end{aligned}
$$

The only way for (22) and (23) to be consistent with the second inequality of (18) is if $a_{2 k}=0 \quad(k=3$, . . . , n)

Continuing in this way, one sees easily that $i \neq k$ implies $a_{i k}=0$; hence, $A$ is normal.

The authors thank J. Todd and H. Wielandt for helpful comments.

[1] O. Toeplitz, Der algebraische Analogon zu einen Satze von Fejér, Math. Z. 2, 187-197 (1918).

[2] G. Frobenius, Über vertauschbare Matrizen, Sitzber. preuss. Ảkad. Wiss. 601-614 (1896).

[3] M. P. Drazin, J. W. Dungey, and K. W. Gruenberg, Some theorems on commutative matrices, J. London Math. Soc. 26, 221-228 (1951)

[4] N. Wiegmann, Pairs of matrices with property L, Proc. Am. Math. Soc. 4, 35-36 (1953).

[5] H. Wielandt, Pairs of matrices with property L, J Research NBS 51 (1953) RP2436.

[6] W. V. Parker, The characteristic roots of matrices, Duke Math. J. 12, 519-526 (1945).

[7] G. H. Hardy, J. E. Littlewood, and G. Pólya, Inequalities, p. 261 (Cambridge Univ. Press, 1934).

[8] H. Weyl, Inequalities between the two kinds of eigenvalues of a linear transformation, Proc. Nat. Acad. Sci. U. S. 35, 408-411 (1949).

[9] K. Fan, On a theorem of Weyl concerning eigenvalues of linear transformations I, Proc. Nat. Acad. Sci. U. S. 35, 652-655 (1949).

Washington, July 28, 1953. 